Monatsschrift für Geburtshülfe u. Gynäkologie 1904;20:I-VI

\title{
Contents, Vol. 20, 1904
}

Inhalts $\bullet$ Verzeichnis.

Opiglnalarbeiten. Seite

Anufrief, A. A., Zur Kasuistik des primäven Tuben-

carcinoms 753

Baisch, K., Hyperemesis und Ptyalismus in der Graviditat 47

Bamberg, G., Incarceration cystisch degenerierter Ovarien

bei Blasenmole. (Hierzu Taf. XVIII) 359

Boesebeek, George, Ein Beitrag zur Ovarialgravidität 613

Brejter, Rud., Ein Beitrag zur Aetiologie der sogen.

„Fistulae cervico-vaginales laqueaticae” 780

v. Bylicki, Ladislaus, Ueber eine Methode, den geraden Beckeneingangsdurchniesser mittelst

einer Skala von Winkelhebeln unmittelbar zu messen 441

Cramer, H., Ueber abdominelle Blasenverlagerung bei

Cystocele 1124

Czerwenka, Karl, Uterus duplex separatus cum Vagina

dupl. separata (Uterus didelpliys) mit Garcinom der

linken Portio 1065

Daniel, B., Kongenitale Nierenanomalien undMissbildungen

der weiblichen Geschlechtsorgane 678

Engels, Einige Bemerkungen zu den Arbeiten „Weitere

Beiträge zur Händedesinfektion” von Dr.R. Scliaeffer 246

d'Erchia, Floi enzo, Beitrag zum Studium des schwangeren

und kreissenden Uterus. (Hierzu Taf. I-VI) . . 1

- - Totale und partielle Uterusexstirpation 841

Foges, Arthur, Ueber die Erfolge der Septumnaht bei

Prolapsoperationen 797

Freund, Hermann W., Die Veränderungen des Nasen-

Rachenraums bei Schwangeren, Gebärenden und

Wöchnerinnen 210, 383

Fro name, Kasuistiscber Beitrag zum Ausgangspunkt gut-

artiger Geschwülste in den grossen Labien .... 961

Fiith, F., Berichtigung und Bemerkung zur Arbeit

Schaeffers über weitere Beiträge zur Hände

desinfektion 241

Graefe, M., Zur Frage der Aetiologie der Hyperemesis

gravidarum

$\mathrm{H}$ alb an, J., Ueber Phlebektasien des graviden Uterus und

ihre klinische Bedeutung 313

Hammersclilag, Die Eklampsie in Ostpreussen . . . 475 
Heinsius, Fritz, Ueber die Beziehungen zwischen kind-lichen und mütterlichen Elementen bei ektopischer Graviditat und ihr Yerhalten nach dem Fruchttode 556

Hengge, Anton, Zum Mechanismus und zur Behandlung

frischer Scheiden-Dammverletzungen ...... 167

Kadyi, Josef von, Der Vorfall der Scheide und der Gebärmutter während der Geburt 1118

i $\gamma$

Keitler, Heinrich, Ueber das anatomische undfimktionelle

Verhalten der belassenen Ovarien nach Exstirpation

des Uterus 686

Kermauner, Fritz, Beiträge zur Anatomie der Tuben-

schwangerschaft 634

Kreidl, Alois, und Ludwig Mandl, Experimentelle Bei

träge zur Lehre von der Absonderung und Ent-

leerung des Harnes im fötalen Leben 910

Kroemer, Paul, Die Prophylaxe der Wochenbetts-

Morbidität in der Schwangerschaft 178

- - Erfolge und Aussicliten der künstlichen F $\pi$ ili-

geburt 901

Kroenig, B., Sublamin als Hände-Desinfiziens .... 62

Liek, Erwin, Beitrag zur Statistik des inoperablen Uteruscarcinoms 227

Linck, Alfred, Ein Fall von primärer Bauchhöhlen-

schwangerschaft .

Mandl, Ludwig s. Kreidl u. M.

Martin, A., Zu den Myomfragen 1130

Müllerheim, R., Ueber die Anwendiing der Kopfzange

bei Beckenendlage 323

Natanson, Hugo, Zur Anatomie und Entwicklungsgeschichte des Uterus unicornis. (Hierzu Taf. XXI

u. XXII) П П95

Neugebauer, Fr. von, Drei interessante Beobachtungen

analoger Missbildung (Hernia funiculi umbilicalis) 1219

Offer geld, Beitrag zur Kenntnis der urämischenLähmungen 522

Opitz, Erich, Umschriebene Fremdkörper-Peritonitis an

der Blase, eine Metastase eines Ovarialtumors vor-

täuschend 966

Papaïoannou, ïh. L., Zur Kenntnis der endothelialen

und metastatischeri Ovarialtumoren. (Hierzu Taf. XII

bis XIV) 802

Pichler, Rudolf, Ein Fall von pigmentierten Schwanger-

schaftsstreifen. (Hierzu Taf. XX) 1089

Pollak, Emil, Weitere Beiträge zur Technik der mecha-nischen Dilatation mit spezieller

Berücksichtigung ihrer Erfolge in der Eklampsiebehandlung . . 335, 951 
Rosenstein, Paul, Anatomische Untersuchungen über den Infektionsweg bei der Genitaltuberkulose des Weibes.

(Hierzu Taf. XV-XYП) 366, 996

Rothe, H, Der Verschluss komplizierter Blasenscheidenfisteln nach Küstner $\quad .790$

Ruge, Carl, Ueber die puerperale Blase und die puerperale Ischurie 537

Rühl, W., Kasuistischer Beitrag zur Indikation der $\mathrm{Au}^{1 / 8} \mathbf{8}^{-}$ schaltung der Bauchpresse während der Geburt . . 468 Santi, E., Die Pathologie des Corpus luteum. (Hierzu Taf. VII-XI) 76, 143

$-\mathrm{V}-$

Simon, Martin E., Untersuchungen über den Gefrierpunkt des Urins während Schwaugerschaft, Geburt und Wochenbett. (Hierzu Taf. XIX) 452

Sitsinsky, A., Die Behandlung der septischen Wochenbetterkrankungen in der Gebärmutterhöhle .... 640

Sonnenfeld, Julius, Klinische Erfahrungen bei Placenta praevia mit besonderer Berücksichtigung des späteren Befindens der Patientinnen 1096

Stolz, Max, Die subkutane Paraftininjektion Gersunys und ihre Verwertung in der Gynäkologie 1238

Weber, Arthur, Die Histologie des Eierstockes imKlimakterium 973

Wladimiroff, W., Zur Trage über das Schicksal der subserosen Fibromyome 761

Wolkowitsen, Nicolai, Eine Methode der Wiederherstellung der weiblichen Harnröhre mit gleichzeitiger Beseitigung einer Blasen-Scheidenfistel. (Hier zu Taf. XXIII) 1253

Zaborszky, Stefan von, Hyperemesis gravidarum . . 39

Zangemeister, W., Ueber Blasensteine 195

Beriehte und Refepate.

Ehrenfest, Hugo, und Fred Taussig, Quartalsbericht über die gynäkologische Literatur der Vereinigten Staaten (II. Semester 1903) 1282

Hartz, A., Neuere Arbeiten über die mesonephrischen Geschwülste 400

Lecène, P., Bericht über die französische geburtshülflichgynäkologische Literatur (I. Semester 1904) . . 1147

Le Maire, M., Sammelbericht über die geburtshülflich-gynäkologische Literatur Dänemarks 1903 ...... 410

1904 (I. Semester). 1142

Mathes, R., Die Eklampsie als Yergiftung 1268

Monchy, M. M. de, Bericht über die geburtshülfliche und gynäkologische Literatur in Holland. III. Quartal 1903101 
IV. Quartal 1903851

Neugebauer, Franz von, Bericht über die polnische geburtshülflich-gynäkologische Literatur der letzten drei Quartale $1902 \quad 105$

Nicholson, Oliphant, Bericht über die englische geburts

hülflich-gynäkologische Literatur $\quad 847$

Taussig, Fred, s. Ehrenfest u. T.

Toff, E., Sammelbericht über im Jahre 1903 in Rumänien

veröffentlichte Arbeiten gynäkologischen und geburts-

hülflichen Inhalts $\quad .855$

Törngren, Adolf, Bericht über die finländische gynä-

kologisch-geburtshülfliche Literatur(П. Semester 1903) Ill

76. Versammlung deutscher Naturforscher und

Aerzte in Breslau 1026

$-\mathrm{VI}-$

Personalien und Tag·esnachrichten.

Personalien und Tagesnachrichten . . 114, 252, 418, 1285

Vereins- und Literaturbeilage.

Opigrinalsitzung'sberiehte aus greburtshül ich-g $\cdot$ ynäkologischen Gesellschaften und Vepeinen.

Gynäkologische Gesellscliaft in Breslau 282, 419

MittelrheiniscL·e Gesellschaft für Geburtshülfe und Gynä

kologie $\quad 273,1151$

Gynäkologische Gesellschaft in München 121, 865

Niederrbeinisch-westfälische Gesellschaft für Geburtshülfe

und Gynäkologie $\quad 886$

Ost- und Westpreussische Gesellschaft für Gynäkologie . 254 Pommersche gynäkologische

Gesellschaft .... 115, 1166 RussischeGesellschaft fürGeburtshülfe und Gynäkologie 1173, 1286

Refepate aus g·ebuFtshülflich-g·ynäkolog·ischen Gesellschaften.

Referate aus geburtshülflich-gynäkologischen Ge

sellschaften 129, 299, 426, 1175, 1299

Gebuptshûlf lieh $-\mathrm{g} \cdot \mathrm{ynäkologische} \mathrm{Mitteilungren} \mathrm{aus} \mathrm{andepen} \mathrm{Gesellschaften} \mathrm{und} \mathrm{Vepeinen.}$

Geburtshülflich-gynäkologische Mitteilungen aus anderen Gesellschaften und Vereinen 132,

303,

431, 1186, 1301 Buchanzeig·en.

Abel, Karl, Vaginale und abdominale Operationen . 308

Burger, 0., s. Mandl und B.

Dohrn, Rud., Geschichte der Geburtshülfe der Neuzeit . 133 Knapp, Ludwig, Der Scheintod der Neugeborenen . . 1189 Mandl, L., und 0. Burger, Die biologische Bedeutung

der Eierstöcke nach Entfernung der Gebärmutter . 432 Martin, A., Diagnostik der

Bauchgeschwülste .... 304 Mitteilungen aus der gynäkologischen Klinik des Prof.

Dr. Otto Engström-Helsingfors. Bd. V. H. 1-3 . 134 Münzer, Histogenese

undPathogenesedesChorioepithelioma

nialígnum $\quad .137$

Stevens, Thos. G., The fate of the ovum and Graefian

follicle in praemenstrual life 434 
Stoeckel, Walter, Die Cystoskopie des Gynäkologen . 900 Stratz, C. H., Die Entwicklung der menschlichen Keim-

blase 305

Litepatupverzeichnis.

Literaturverzeichnis . . 138, 309, 435, 1060, 1190, 1302 